\title{
Astrometric observations of neutron stars
}

\author{
S. Chatterjee \\ School of Physics, The University of Sydney, \\ NSW 2006, Australia \\ email: S.Chatterjee@physics.usyd.edu.au
}

\begin{abstract}
Precision astrometry can yield model-independent distances and velocities for neutron stars. Such measurements can be exploited, for example, to locate neutron star birth sites, establish reference frame ties, model the Galactic electron density distribution, and constrain the astrophysics of supernova explosions. As a case study, I discuss recent some parallax and proper motion measurements, and their scientific implications for supernova core collapse and the velocities of ordinary pulsars versus magnetars. I also outline the calibration techniques that are enabling sub-milliarcsecond astrometry of neutron stars with VLBI. In the short term, systematic surveys and high sensitivity on very long baselines will produce ongoing science dividends from precision astrometry at radio wavelengths. In the longer term, new technology such as focal plane arrays, new telescopes such as the Square Kilometre Array, and synergy with new instruments such as Gaia, LSST, and GLAST, all hold great promise in an upcoming era of microarcsecond astrometry.
\end{abstract}

Keywords. astrometry, techniques: interferometric, techniques: high angular resolution, stars: neutron, pulsars: general

\section{Introduction}

Neutron stars are exotic laboratories for some of the most extreme physics in the Universe. Astrometric observations of neutron stars (NS) can be exploited to constrain their origins, evolution, and environments, as well as to establish meaningful constraints on nuclear physics, particle physics, and theories of gravitation.

The basic observable, as in most other astrometric applications, is the position of the NS. For individual objects, rather than an ensemble of stars over the entire sky, such measurements are necessarily relative in nature, but absolute positions can be inferred from measurements relative to sources that define the ICRF (Ma et al. 1998). Over time, repeated measurements of the position $\vec{\theta}$ allow a proper motion $\vec{\mu}$ to be derived. For a precise proper motion, the primary consideration is a long time baseline, limited by the stability of the reference frame and the variability of the frame-defining sources. Finally, with enough astrometric precision, a trigonometric parallax $\pi$ may also be measurable for NS. The primary consideration for such measurements is appropriate sampling over the Earth's orbital phase, not just a long time baseline.

Neutron stars emit over a broad range of wavelengths, and astrometric observations of NS have been conducted at wavelengths from radio to X-rays. For example, Kaplan et al. (2007) have used optical observations with the Hubble Space Telescope to mea-

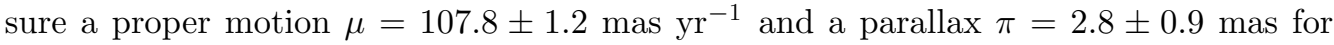
RX J0720.4-3125. At X-ray wavelengths, the resolution that can be achieved with the current generation of telescopes is a limiting factor, but Winkler \& Petre (2007) have used the Chandra X-ray Observatory to measure a proper motion of $165 \pm 25 \mathrm{mas} \mathrm{yr}^{-1}$ for the RX J0822-4300, in the center of the Puppis A supernova remnant. 


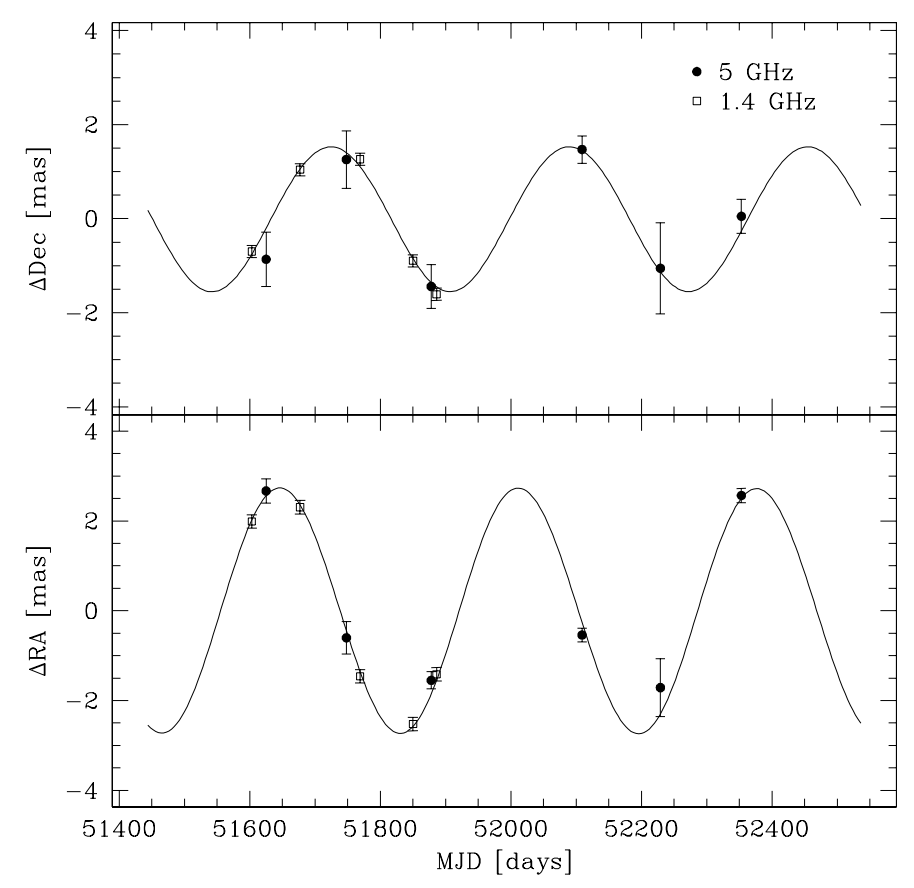

Figure 1. The parallax signature of PSR B1929+10 in right ascension and declination, after subtracting the best-fit proper motion from the astrometric positions measured with the VLBA at $5 \mathrm{GHz}$ (closed circles; Chatterjee et al. 2004) and $1.4 \mathrm{GHz}$ (open squares; Brisken et al. 2002). Sinusoids corresponding to the best fit parallax $\pi=2.77$ mas are overplotted. Figure adapted from Chatterjee et al. (2004).

The majority of known NS are radio pulsars, and pulse timing is routinely used to refine their positions and proper motions. A subset of recycled ("millisecond") pulsars have rotation rates that are stable enough to permit sub-milliarcsecond astrometry based on pulse time of arrival. For example, van Straten et al. (2001) measure the general relativistic Shapiro delay for the binary pulsar J0437-4715, as well as a precise proper motion and parallax, based on pulse timing at Parkes. However, most pulsars do not have such stable rotation, particularly when they are young, and Very Long Baseline interferometry (VLBI) has usually been utilized to determine their astrometric parameters. Such efforts have a long history (e.g., Gwinn et al. 1986), but have become much more feasible with the Very Long Baseline Array (VLBA), which provides full-time, dedicated VLBI capabilities with identical antennas, allowing good control of systematic errors and leading to many recent parallax measurements (e.g., Fomalont et al. 1999; Brisken et al. 2000; Chatterjee et al. 2001; Brisken et al. 2002; Chatterjee et al. 2004, see Figure 1).

\section{Scientific applications of neutron star astrometry}

Precise and accurate measurements of the position, proper motion, and parallax of NS can be exploited to address a variety of scientific questions.

Position: Measuring the position of an object in two different coordinate frames permits the very fundamental operation of tying the two reference frames together. Since they are compact sources and are accessible to astrometry with different techniques and at different wavelengths, NS are particularly well suited to such reference frame ties. 
Specifically, pulse timing provides radio pulsar positions in the Solar system reference frame, while VLBI measurements are tied to the distant quasars. Thus, simply measuring precise positions for recycled pulsars enables fundamental reference frame ties between the Solar system and the extragalactic ICRF (e.g. Bartel et al. 1996). A few pulsars also have optical counterparts, and PSR J0437-4715, for example, may provide a frame-tie between the optical reference frame and the ICRF for the Space Interferometry Mission (SIM).

Proper Motion: Precise proper motions for NS allow them to be traced back to their birth sites in massive stellar clusters, and to associations with runaway stars (Hoogerwerf et al. 2000; Vlemmings et al. 2004; Chatterjee et al. 2005, see Figure 2). For very young objects, associations with their progenitor supernova remnants can be verified or refuted, leading to independent age estimates for both the NS and the supernova remnant itself (e.g., Gaensler \& Frail 2000; Migliazzo et al. 2002; Blazek et al. 2006). The rotation of such young pulsars is typically not stable enough for astrometry via pulse timing, and interferometry is thus required. Combined with estimates for their distances, the proper motions of NS also lead to velocity estimates. The high velocity tail of the distribution implies that large kicks are imparted to proto-neutron stars during core collapse, as discussed further below.

Parallax: When a parallax measurement is possible, it provides a model independent estimate for the distance and velocity of the neutron star. Each such measurement calibrates global models of the Galactic electron density (Taylor \& Cordes 1993; Cordes \& Lazio 2002), thus improving distance estimates from pulse dispersion measure for the rest of the radio pulsar population, as well as probing the distribution of electron density in the local interstellar medium (e.g., Toscano et al. 1999).

Observed thermal radiation from the NS surface can be used, in combination with a precise distance, to constrain the 'size' of the photosphere, the NS radius, and thus the Equation of State of matter at extreme pressures and densities (Yakovlev \& Pethick 2004; Lattimer \& Prakash 2004). For radio pulsars, uncertainties in the magnetospheric emission restricts such an exercise to very young and hot objects (e.g., PSR B0656+14, Brisken et al. 2003), while isolated NS which are X-ray bright and radio quiet pose a challenge for optical astrometry (e.g., RX J1856.5-3754, Kaplan et al. 2002; Walter \& Lattimer 2002).

\section{Case study: the astrophysics of pulsar kicks}

Since their discovery, radio pulsars have been known to form a high velocity population. Statistical studies of the velocity distribution of ordinary young radio pulsars (Lyne \& Lorimer 1994; Arzoumanian et al. 2002; Hobbs et al. 2005; Faucher-Giguère \& Kaspi 2006) yield mean three-dimensional population velocities of $300-500 \mathrm{~km} \mathrm{~s}^{-1}$, while the existence of objects such as PSR B2224+65 (the Guitar Nebula pulsar, with a transverse velocity $\sim 800-1600 \mathrm{~km} \mathrm{~s}^{-1}$ ) demonstrates the presence of a long high-velocity tail (Cordes et al. 1993; Chatterjee \& Cordes 2004).

A number of physical mechanisms have been suggested to account for the high speeds, including the disruption of binaries through mass loss in supernovae (Blaauw 1961; Iben \& Tutukov 1996) and the electromagnetic rocket effect (Harrison \& Tademaru 1975), but it now appears likely that NS gain a large part of their observed velocity from a "birth kick" that involves asymmetries in the natal supernova explosion (Lai et al. 2001). The nature of the asymmetry remains unclear: while hydrodynamic or convective instabilities are plausible (Burrows \& Hayes 1996; Janka \& Mueller 1996), more exotic mechanisms 


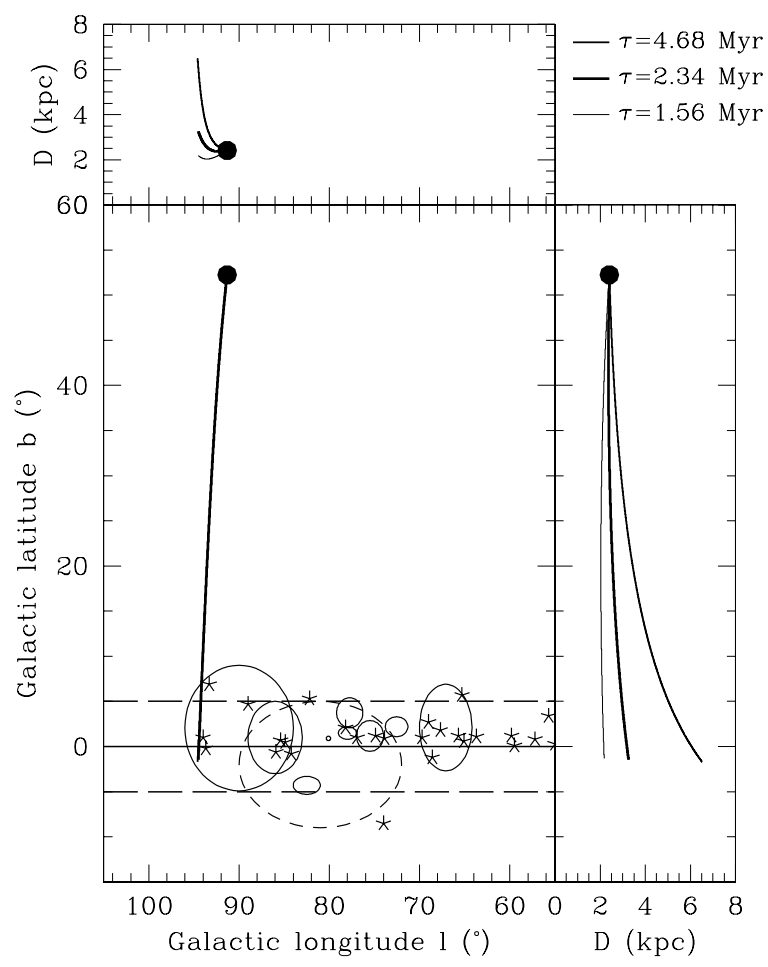

Figure 2. Possible orbits for PSR B1508+55, traced back in the Galactic potential. The solid dot denotes the current pulsar position and the thick solid line the path it has followed for an age $\tau=2.34 \mathrm{Myr}$ (the spindown age, with braking index $n=3$ ) and a radial velocity $v_{r}=200 \mathrm{~km} \mathrm{~s}^{-1}$. Other possible orbits are shown (thinner lines) for a range of ages and radial velocities which lead to birth sites in the Galactic plane $(\tau=4.69 \mathrm{Myr}$ for $n=4$ and $v_{r}=-300 \mathrm{~km} \mathrm{~s}^{-1}, \tau=1.56 \mathrm{Myr}$ for $n=2$ with $\left.v_{r}=700 \mathrm{~km} \mathrm{~s}^{-1}\right)$. Also indicated are the Cygnus superbubble (2 kpc away, dashed ellipse), the Cygnus OB associations (solid ellipses), and Galactic supernova remnants identified in this region. The solid horizontal line is the Galactic plane and the horizontal dashed lines indicate the pulsar birth scale height from Arzoumanian et al. (2002) at the distance of the Cygnus superbubble. At its characteristic age, the pulsar can thus be traced back to a birth site in the Galactic plane and near massive stars in $\mathrm{OB}$ associations which are plausible NS progenitors. Figure adapted from Chatterjee et al. (2005).

such as asymmetric neutrino emission in the presence of strong magnetic fields cannot be ruled out (Arras \& Lai 1999).

The high velocity tail of the pulsar birth velocity distribution is of particular interest, since these kicks impose the most stringent constraints on mechanisms for supernova core collapse. Currently, two-dimensional hydrodynamic models can provide a supernova detonation, a NS kick, and an asymmetric supernova remnant by invoking neutrino energy deposition (Scheck et al. 2004, 2006). However, the first full three-dimensional simulations of supernovae (Fryer 2004) have difficulty in producing the required magnitude of birth kicks, possibly implying the presence of extremely high magnetic fields $>10^{15} \mathrm{G}$ (Arras \& Lai 1999) or some combination of the above effects (Socrates et al. 2005) in driving kicks.

A magnetic field-driven kick mechanism immediately suggests the possibility that the population of magnetars (which have magnetic field decay as their primary energy source 
and usually emit no detectable radio pulsations) should have much larger kick velocities than the pulsar population. Indeed, in proposing the existence of magnetars, Duncan \& Thompson (1992) suggested that an initial short birth period might be responsible for the generation of the high magnetar fields through an efficient large scale dynamo, and that these high magnetic fields could mediate asymmetric neutrino emission at birth, resulting in extreme space velocities (Thompson \& Duncan 1993).

Astrometric observations have established significant constraints on these questions. Specifically, a VLBA proper motion and parallax for PSR B1508+55 (Chatterjee et al. $2005)$ provides a model independent transverse velocity estimate $V_{\perp}=1083_{-90}^{+103} \mathrm{~km} \mathrm{~s}^{-1}$, posing a significant challenge to those supernova core collapse simulations which do not provide large kicks. The extremely ordinary spin and spindown characteristics of the pulsar also challenge exotic kick scenarios that require extreme magnetic fields for high birth velocities.

Measuring the velocity of a population of magnetars and comparing them to the radio pulsar population would allow a clear test of magnetic field-driven kick mechanisms, and efforts to make such measurements are underway using X-ray and infra-red observations. In the meantime, the discovery of radio pulses from the magnetar XTE J1810-197 (Camilo et al. 2006), while posing a variety of theoretical challenges to ideas about magnetars, also allowed a rapid proper motion determination (Helfand et al. 2007) using the much higher angular resolutions offered by the VLBA. Helfand et al. (2007) find $V_{\perp} \sim 212 \pm 35 \mathrm{~km} \mathrm{~s}^{-1}$ for a distance of $3.5 \pm 0.5 \mathrm{kpc}$. Only the transverse velocity is measured, and for only a single magnetar, but with those caveats, the estimate is completely consistent with the population velocity of ordinary pulsars.

Finally, if birth kicks also influence the initial spin rate of the NS core (Spruit \& Phinney 1998), then the spin and kinematic histories of each NS would be closely linked, but it is unknown whether or not such a link exists (Deshpande et al. 1999; Romani \& Ng 2003). The Crab pulsar has a well defined symmetry axis, traced by the X-ray jet-torus structure and the optical equatorial wisps (Hester et al. 2002; Ng \& Romani 2004), and the axis is naturally associated with the NS spin, since every other vector is rotation averaged. Kaplan et al. (2008) use archival HST observations to determine a precise proper motion for

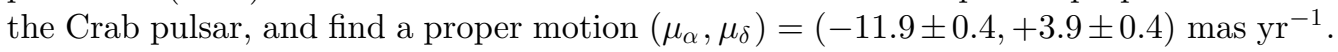
However, in comparing the proper motion vector to the position angle of the spin axis, they find that the unknown motion of the progenitor star contributes a systematic uncertainty that dominates the statistical errors, leading to an estimate of the misalignment of $14^{\circ} \pm 2^{\circ} \pm 9^{\circ}$, consistent with a broad range of values, including zero. Thus, the alignment of NS spin axes with their kick vectors is better tested over a statistical ensemble rather than with individual objects.

\section{Calibration techniques and future directions}

As illustrated above, astrometry can bring powerful constraints to bear on a variety of scientific questions about neutron stars, their origins, astrophysics, evolution, and environments. The primary obstacle is the difficulty of such astrometric observations. At optical, infra-red, and X-ray wavelengths, only a few NS can be observed and the required resolution for high-precision astrometry is difficult to attain.

At radio wavelengths, many pulsars (and a couple of magnetars) can be observed with VLBI. However, not only are most pulsars faint, but the most interesting categories, namely the youngest pulsars and the recycled ones, appear to be disproportionately faint. Pulsar gating (which accumulates signal only during the predicted on-pulse periods for pulsars) is now routinely employed to boost the signal to noise ratios for VLBA 

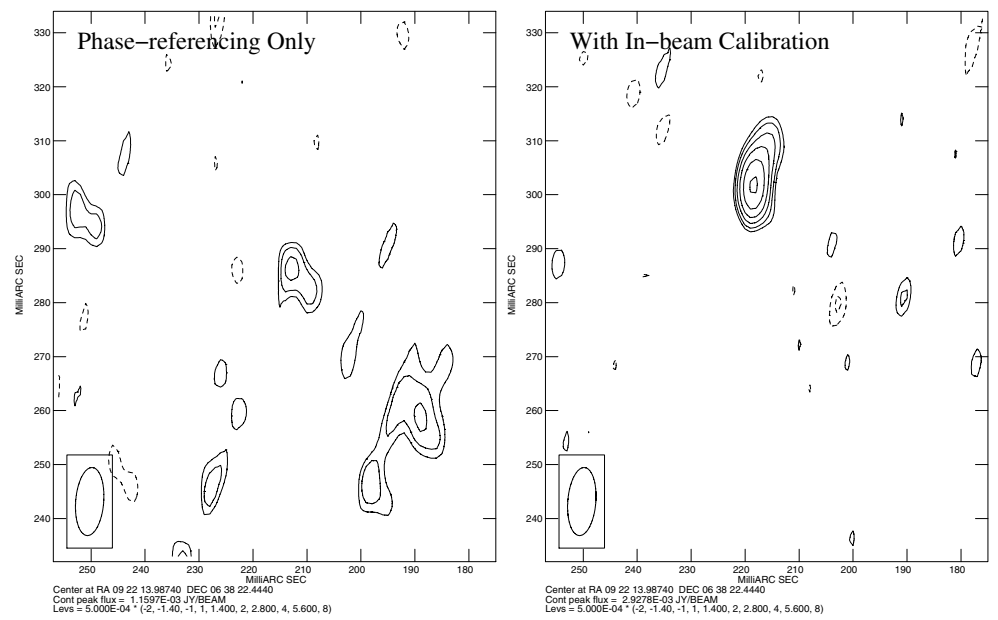

Figure 3. VLBA images of PSR B0919+06 at $1.4 \mathrm{GHz}$ (left) after phase referencing to a calibrator $\sim 4^{\circ}$ away, and (right) after in-beam calibration with a calibrator $12^{\prime}$ away, within the primary beam of the antennas. On this occasion, a disturbed ionosphere does not allow useful astrometry with phase referencing alone, but with in-beam calibration, precise astrometric information can be effectively recovered.

astrometric observations. To make matters worse, however, it is not just a lack of sensitivity but systematic errors contributed by the ionosphere and the troposphere which are the primary impediments to sub-milliarcsecond astrometry. Progress has been made, for example, with GPS-based ionospheric calibration schemes. In-beam calibration (Fomalont et al. 1999), using a faint source in the same primary beam as the target source, has also proved to be effective (see Figure 3).

Currently, large dishes (like the Arecibo and Green Bank telescopes) are being employed in concert with the VLBA to boost sensitivity for pulsar astrometry, though such high-sensitivity arrays pose calibration challenges. In the Southern hemisphere, the Long Baseline Array is conducting astrometric observations, though the available baseline lengths are currently limited. Further in the future, focal plane feed arrays have been identified as a key technology for instruments now on the drawing board, including the Square Kilometer Array (SKA). By effectively offering much larger fields of view, a focal plane array allows the use of many faint reference sources, thus opening up a large fraction of the pulsar population to high-precision astrometry. It is also noteworthy that telescopes like the SKA will require a significant long baseline component in order to avoid being hopelessly confusion limited by faint sources, and such a long baseline component is ideal for high-sensitivity NS astrometry.

At optical and infra-red wavelengths, adaptive optics, high-sensitivity detectors, and new space-based missions like SIM and Gaia provide significant opportunities for astrometry of faint NS. Meanwhile, LSST, GLAST, and other survey instruments at a wide range of wavelengths will depend on precise astrometric observations to analyze and interpret their results.

\section{Acknowledgements}

I acknowledge a generous travel grant from the organizing committee for this conference, and support from the University of Sydney Postdoctoral Fellowship program. I also acknowledge my collaborators on a variety of projects, including Walter Brisken, 
Jim Cordes, Ed Fomalont, Bryan Gaensler, Miller Goss, David Helfand, David Kaplan, Michael Kramer, Joe Lazio, Andrew Lyne, and Wouter Vlemmings.

\section{References}

Arras, P. \& Lai, D. 1999, ApJ, 519, 745

Arzoumanian, Z., Chernoff, D. F., \& Cordes, J. M. 2002, ApJ, 568, 289

Bartel, N., Chandler, J. F., Ratner, M. I., Shapiro, I. L., Pan, R., \& Cappallo, R. J. 1996, AJ, 112,1690

Blaauw, A. 1961, Bull. Astron. Inst. Netherlands, 15, 265

Blazek, J. A., Gaensler, B. M., Chatterjee, S., van der Swaluw, E., Camilo, F., \& Stappers, B. W. 2006, ApJ, 652, 1523

Brisken, W. F., Benson, J. M., Beasley, A. J., Fomalont, E. B., Goss, W. M., \& Thorsett, S. E. 2000, ApJ, 541, 959

Brisken, W. F., Benson, J. M., Goss, W. M., \& Thorsett, S. E. 2002, ApJ, 571, 906

Brisken, W. F., Thorsett, S. E., Golden, A., \& Goss, W. M. 2003, ApJ Lett., 593, L89

Burrows, A. \& Hayes, J. 1996, Physical Review Letters, 76, 352

Camilo, F., Ransom, S. M., Halpern, J. P., Reynolds, J., Helfand, D. J., Zimmerman, N., \& Sarkissian, J. 2006, Nature, 442, 892

Chatterjee, S. \& Cordes, J. M. 2004, ApJ Lett., 600, L51

Chatterjee, S., Cordes, J. M., Lazio, T. J. W., Goss, W. M., Fomalont, E. B., \& Benson, J. M. 2001, ApJ, 550, 287

Chatterjee, S., Cordes, J. M., Vlemmings, W. H. T., Arzoumanian, Z., Goss, W. M., \& Lazio, T. J. W. 2004, ApJ, 604, 339

Chatterjee, S., Vlemmings, W. H. T., Brisken, W. F., Lazio, T. J. W., Cordes, J. M., Goss, W. M., Thorsett, S. E., Fomalont, E. B., Lyne, A. G., \& Kramer, M. 2005, ApJ Lett., 630, L61

Cordes, J. M. \& Lazio, T. J. W. 2002, ArXiv e-print, astro-ph/0207156

Cordes, J. M., Romani, R. W., \& Lundgren, S. C. 1993, Nature, 362, 133

Deshpande, A. A., Ramachandran, R., \& Radhakrishnan, V. 1999, A\&\&A., 351, 195

Duncan, R. C. \& Thompson, C. 1992, ApJ Lett., 392, L9

Faucher-Giguère, C.-A. \& Kaspi, V. M. 2006, ApJ, 643, 332

Fomalont, E. B., Goss, W. M., Beasley, A. J., \& Chatterjee, S. 1999, AJ, 117, 3025

Fryer, C. L. 2004, ApJ Lett., 601, L175

Gaensler, B. M. \& Frail, D. A. 2000, Nature, 406, 158

Gwinn, C. R., Taylor, J. H., Weisberg, J. M., \& Rawley, L. A. 1986, AJ, 91, 338

Harrison, E. R. \& Tademaru, E. 1975, ApJ, 201, 447

Helfand, D. J., Chatterjee, S., Brisken, W. F., Camilo, F., Reynolds, J., van Kerkwijk, M. H., Halpern, J. P., \& Ransom, S. M. 2007, ApJ, 662, 1198

Hester, J. J., Mori, K., Burrows, D., Gallagher, J. S., Graham, J. R., Halverson, M., Kader, A., Michel, F. C., \& Scowen, P. 2002, ApJ Lett., 577, L49

Hobbs, G., Lorimer, D. R., Lyne, A. G., \& Kramer, M. 2005, MNRAS, 360, 974

Hoogerwerf, R., de Bruijne, J. H. J., \& de Zeeuw, P. T. 2000, ApJ Lett., 544, L133

Iben, I. J. \& Tutukov, A. V. 1996, ApJ, 456, 738

Janka, H.-T. \& Mueller, E. 1996, A\&\&A., 306, 167

Kaplan, D. L., Chatterjee, S., Gaensler, B. M., \& Anderson, J. 2008, ApJ, accepted, ArXiv e-print 0801.1142

Kaplan, D. L., van Kerkwijk, M. H., \& Anderson, J. 2002, ApJ, 571, 447

一. 2007, ApJ, 660, 1428

Lai, D., Chernoff, D. F., \& Cordes, J. M. 2001, ApJ, 549, 1111

Lattimer, J. M. \& Prakash, M. 2004, Science, 304, 536

Lyne, A. G. \& Lorimer, D. R. 1994, Nature, 369, 127

Ma, C., Arias, E. F., Eubanks, T. M., Fey, A. L., Gontier, A.-M., Jacobs, C. S., Sovers, O. J., Archinal, B. A., \& Charlot, P. 1998, AJ, 116, 516

Migliazzo, J. M., Gaensler, B. M., Backer, D. C., Stappers, B. W., van der Swaluw, E., \& Strom, R. G. 2002, ApJ Lett., 567, L141 
Ng, C.-Y. \& Romani, R. W. 2004, ApJ, 601, 479

Romani, R. W. \& Ng, C.-Y. 2003, ApJ Lett., 585, L41

Scheck, L., Kifonidis, K., Janka, H.-T., \& Müller, E. 2006, A\&A., 457, 963

Scheck, L., Plewa, T., Janka, H.-T., Kifonidis, K., \& Müller, E. 2004, Physical Review Letters, 92, 011103

Socrates, A., Blaes, O., Hungerford, A., \& Fryer, C. L. 2005, ApJ, 632, 531

Spruit, H. C. \& Phinney, E. S. 1998, Nature, 393, 139

Taylor, J. H. \& Cordes, J. M. 1993, ApJ, 411, 674

Thompson, C. \& Duncan, R. C. 1993, ApJ, 408, 194

Toscano, M., Britton, M. C., Manchester, R. N., Bailes, M., Sandhu, J. S., Kulkarni, S. R., \& Anderson, S. B. 1999, ApJ Lett., 523, L171

van Straten, W., Bailes, M., Britton, M., Kulkarni, S. R., Anderson, S. B., Manchester, R. N., \& Sarkissian, J. 2001, Nature, 412, 158

Vlemmings, W. H. T., Cordes, J. M., \& Chatterjee, S. 2004, ApJ, 610, 402

Walter, F. M. \& Lattimer, J. M. 2002, ApJ Lett., 576, L145

Winkler, P. F. \& Petre, R. 2007, ApJ, 670, 635

Yakovlev, D. G. \& Pethick, C. J. 2004, Ann. Rev. Astron. Astrophys., 42, 169 